\title{
Costoefectividad del régimen combinado de mirabegron/solifenacina en el tratamiento del síndrome de vejiga hiperactiva en Colombia
}

\section{Cost-effectiveness of the Combined Regimen of Mirabegron/Solifenacin in the Treatment of Overactive Bladder Syndrome in Colombia}

\author{
Pieralessandro Lasalvia ${ }^{1,2}$ Yaneth Gil-Rojas ${ }^{1}$ Fabián Hernández ${ }^{1 \oplus} \quad$ Camilo Castañeda-Cardona $^{1}$ \\ ${ }^{1}$ Departamento de Estudios Económicos, Neuroeconomix, Bogotá,
Colombia \\ ${ }^{2}$ Departamento de Epidemiología Clínica y Bioestadística, Facultad de \\ Medicina, Pontificia Universidad Javeriana, Bogotá, Colombia \\ Dirección para correspondencia Yaneth Gil Rojas, MSc, \\ Departamento de Estudios Económicos, Neuroeconomix, Calle 45 No. \\ 9-42, Bogotá, Colombia (e-mail: yaneth@neuroeconomix.com).
}

Urol Colomb 2021;30(4):e277-e285.

\section{Resumen}

Palabras Clave

- costoefectividad

- mirabegron

- solifenacina

- terapia combinada

- onabotulinumtoxina A

- vejiga urinaria hiperactiva
Objetivo Evaluar la costoefectividad incremental del régimen combinado de mirabegron/solifenacina en comparación con el uso temprano de toxina botulínica, desde la perspectiva del sistema de salud colombiano, para el tratamiento de adultos con vejiga hiperactiva.

Métodos Se empleó un modelo de Markov en que se comparan dos secuencias de tratamiento, una con y otra sin mirabegron/solifenacina, para evaluar la costoefectividad en un horizonte temporal de cinco años. Debido a la perspectiva de análisis, sólo se tuvieron en cuenta los costos médicos directos. La eficacia del tratamiento evaluado y su comparador fue medida en términos de la reducción de episodios diarios de incontinencia y de la frecuencia de micciones. Los costos fueron expresados en pesos colombianos de 2019, y se aplicó una tasa de descuento de $5 \%$ tanto para desenlaces como para costos.

Resultados Para el caso base, el costo del tratamiento en la secuencia que incluye mirabegron/solifenacina fue mayor, pero generó un mayor número de años de vida ajustados por calidad, y así e obtuvo una razón de costoefectividad incremental de $\$ 13.637,184$ si se considera el desenlace de reducción de episodios diarios de incontinencia de $50 \%$, y de $\$ 29.313,848$ si se considera el del $100 \%$.

Conclusiones De acuerdo con los resultados de esta evaluación, para un horizonte de análisis de cinco años, la secuencia de tratamiento con mirabegron/solifenacina es una alternativa costoefectiva, si se considera un umbral de disposición a pagar de tres veces el producto interno bruto (PIB) per cápita. recibido

12 de enero de 2021

aceptado

04 de agosto de 2021
DOI https://doi.org/

$10.1055 / \mathrm{s}-0041-1740291$

ISSN 0120-789X.

e ISSN 2027-0119.
(C) 2021. Sociedad Colombiana de Urología. All rights reserved. This is an open access article published by Thieme under the terms of the Creative Commons Attribution-NonDerivative-NonCommercial-License, permitting copying and reproduction so long as the original work is given appropriate credit. Contents may not be used for commercial purposes, or adapted, remixed, transformed or built upon. (https://creativecommons.org/ licenses/by-nc-nd/4.0/)

Thieme Revinter Publicações Ltda., Rua do Matoso 170, Rio de Janeiro, RJ, CEP 20270-135, Brazil 


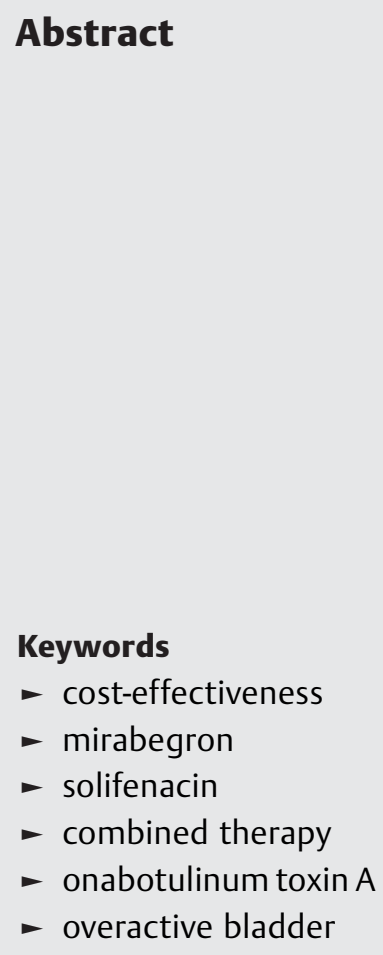

Aim To evaluate the incremental cost-effectiveness of the combined regimen of mirabegron/solifenacin compared with the early use of botulinum toxin, from the perspective of the Colombian health system, for the treatment of adults with overactive bladder.

Methods A Markov model comparing two treatment sequences, one with and one without mirabegron/solifenacin, was used to assess cost-effectiveness over a five-year period. Due to the perspective of the analysis, only direct medical costs were considered. The efficacy of the evaluated treatment and its comparator was measured in terms of the reduction in the daily incontinence episodes and the frequency of micturition. The costs were expressed in Colombian pesos of 2019, and a discount rate of $5 \%$ was applied for both outcomes and costs.

Results For the base case, the cost of the treatment in the sequence that includes mirabegron/solifenacin was higher, but it generated a greater number of qualityadjusted years of life, thus obtaining an incremental cost-effectiveness ratio of $\$ 13,637,184$ when considering the outcome of $50 \%$ of reduction in the daily incontinence episodes, and $\$ 29,313,848$ when considering $100 \%$.

Conclusions According to the results of the present assessment, for a five-year period of analysi, the mirabegron/solifenacin treatment sequence is a cost-effective alternative when considering a threshold of willingness to pay three times the per capita gross domestic product (GDP).

\section{Introducción}

La vejiga hiperactiva $(\mathrm{VH})$ es una condición crónica que tiene un alto impacto en la calidad de vida de los pacientes. ${ }^{1}$ Se caracteriza por urgencia urinaria que, por lo general, se acompaña de frecuencia y nocturia, y pueden o no presentarse episodios de incontinencia urinaria (EIUs) de urgencia. ${ }^{1}$ Aunque la etiología no está bien establecida, se le ha considerado una condición de naturaleza multifactorial, y, entre los factores predisponentes descritos, se encuentran: cambios anatómicos, estilo de vida, y comorbilidades. ${ }^{2,3}$ Entre las causas de naturaleza idiopática está la hiperactividad del detrusor, que da lugar a los síntomas de urgencia y frecuencia, con o sin incontinencia; sin embargo, diversas condiciones neurológicas pueden producir 0 exacerbar la hiperactividad del detrusor. ${ }^{1}$

Debido a la heterogeneidad de los síntomas y el subreporte por parte de los pacientes, la prevalencia e incidencia es difícil de establecer, pero se estima que afecta aproximadamente $11 \%$ y $16 \%$ de la población adulta, y se incrementa con la edad. ${ }^{1-3}$ En un estudio multicéntrico del año 2010 realizado en Venezuela se reportó una prevalencia de $21 \%$, y en otros estudios conducidos en Latinoamérica se reportaron prevalencias similares, del $23,4 \%$ y $18,9 \%$ en Brasil y Argentina, respectivamente. ${ }^{4}$ En Colombia, la prevalencia de $\mathrm{VH}$ en adultos, estimada en el estudio COBaLT, ${ }^{5}$ es de $31,8 \%$.

Los cambios comportamentales constituyen la primera línea de tratamiento, e incluyen cambios en el estilo de vida y entrenamiento de la vejiga y de los músculos del piso pélvico. $^{1-3}$ Entre las opciones farmacológicas, los agentes antimuscarínicos (oxibutinina, tolterodina, fesoterodina, darifenacina, y solifenacina) han sido ampliamente usados, y se recomiendan como una segunda línea de tratamiento luego de las intervenciones comportamentales. ${ }^{1,3}$ El mirabegron, un agonista adrenérgico $\beta 3$, es otra de las opciones farmacológicas actualmente disponibles. Las inyecciones intravesicales de onabotulinumtoxina A (OBA) se emplean como una tercera línea en pacientes en los que han fallado la primera y segunda líneas de tratamiento, o en los que no son candidatos a medicamentos anticolinérgicos. ${ }^{1}$

La solifenacina y el mirabegron ya se encuentran aprobados en Colombia como monofármacos para el tratamiento de la $\mathrm{VH}, \mathrm{y}$, recientemente, el régimen combinado ha sido aprobado en la misma indicación. ${ }^{6}$ La eficacia y seguridad del régimen combinado $^{7}$ ha sido evaluada en 3 estudios aleatorizados, de doble ciego, de 12 semanas de seguimiento (Symphony, ${ }^{8}$ SYNERGY $^{9}$ y $\left.\mathrm{BESIDE}^{10}\right)$. En estos estudios, la combinación mostró beneficios en términos de reducción del número de micciones en 24 horas y en el número de episodios de incontinencia en comparación con la monoterapia o placebo.

Teniendo en cuenta que la atención de la $\mathrm{VH}$ debe ser priorizada debido a su impacto en términos de calidad de vida y al impacto económico para el sistema de salud, relacionados con la policonsulta, el uso de pañales y otros insumos, el uso de medicamentos, y la necesidad de procedimientos quirúrgicos, ${ }^{1}$ el objetivo de esta investigación fue evaluar, desde la perspectiva del sistema de salud colombiano, la costoefectividad incremental del régimen combinado de mirabegron/solifenacina $(\mathrm{M}+\mathrm{S})$ en el tratamiento de adultos con $\mathrm{VH}$. 


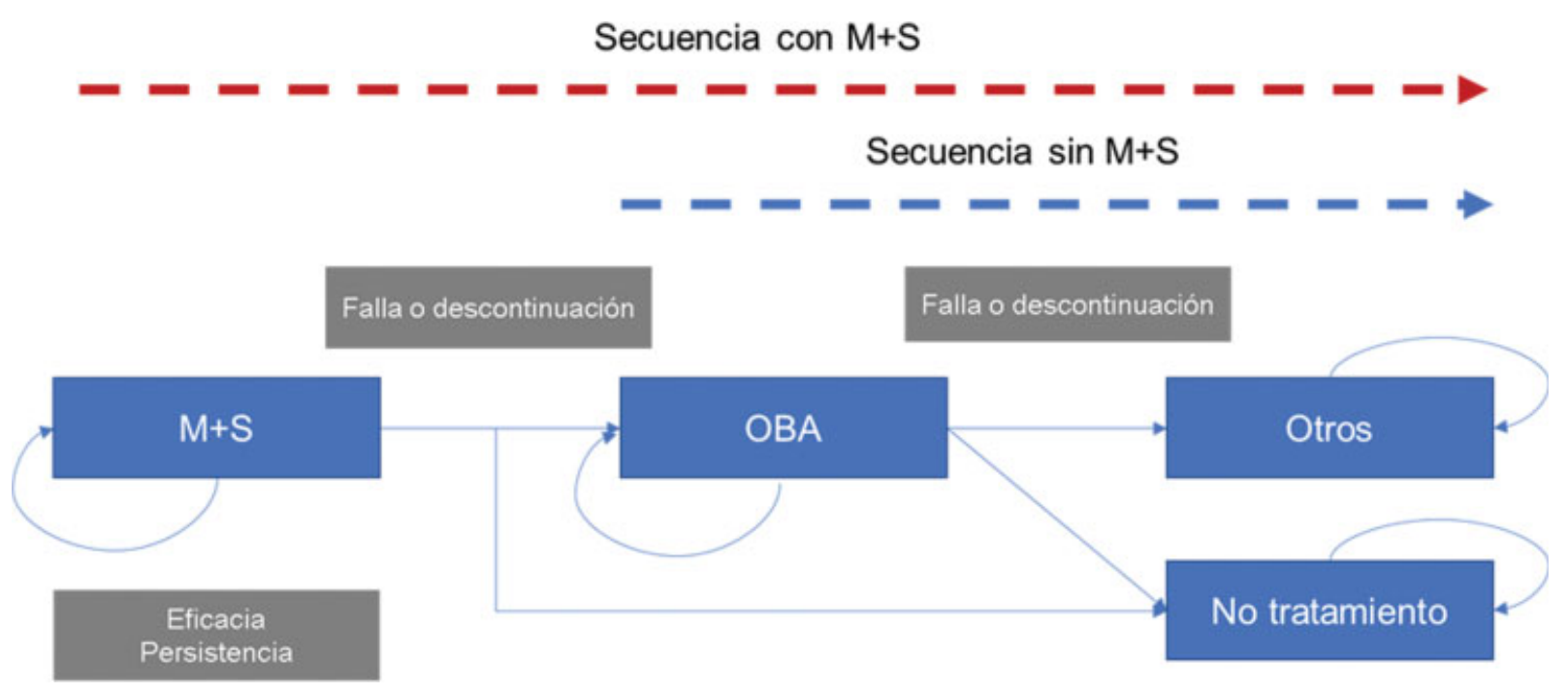

Fig. 1 Estructura del modelo.

\section{Métodos}

Intervención y comparadores: la tecnología evaluada fue la secuencia de tratamiento con $\mathrm{M}+\mathrm{S}$. Para el uso en combinación, la dosis recomendada es de $50 \mathrm{mg}$ de mirabegron y $5 \mathrm{mg}$ de solifenacina, administrados $1 \mathrm{vez}$ al día. ${ }^{11}$ Se consideró un análisis secuencial, por lo que los pacientes en los que que falla el régimen combinado serían candidatos para recibir toxina botulínica. El comparador definido para esta evaluación fue la secuencia de tratamiento farmacológico habitual en los pacientes en los que falla la primera línea de tratamiento, es decir, el inicio de OBA. De OBA se recomiendan 20 inyecciones de $0,5 \mathrm{~mL}$ (concentración recomendada: $10 \mathrm{U} / \mathrm{mL}$ ) para 1 dosis total de 100 unidades/10 mL (máximo: 100 unidades).

Perspectiva, horizonte temporal, y tasa de descuento: La evaluación económica se realizó desde la perspectiva del tercer pagador (sistema de salud colombiano). El horizonte de análisis fue de 5 años, con ciclos mensuales para la medición de los costos y de la efectividad. El horizonte se basó principalmente en lo reportado en otros estudios económicos, ${ }^{13-16}$ y tiene en cuenta que la persistencia de los pacientes con los tratamientos farmacológicos en general es baja. Para el caso base, se aplicó una tasa de descuento de $5 \%$ tanto para los costos como para los desenlaces en salud, de acuerdo con las recomendaciones del Manual del Instituto de Evaluación Tecnológica en Salud (IETS). ${ }^{17}$

Modelo: el modelo fue desarrollado en Excel (Microsoft Corp., Redmond, WA, EEUU) con Visual Basic for Applications (VBA). La población objetivo fueron pacientes con diagnóstico de $\mathrm{VH}$ que no tuvieron una respuesta adecuada con anticolinérgicos o mirabegron empleados en monoterapia. Las características de la población modelada, en particular, los episodios de incontinencia/día y su promedio, y la distribución de los pacientes entre los diferentes niveles de severidad de la incontinencia, se basaron en el estudio SYNERGY. ${ }^{9}$
Se desarrolló un modelo de Markov, que simula el manejo terapéutico de los pacientes en los que fallan los anticolinérgicos en monoterapia. Se evaluaron dos posibles secuencias de tratamiento (-Figura 1). En la secuencia de análisis, los pacientes inician el tratamiento $\mathrm{M}+\mathrm{S}$ y, en cada ciclo, pueden presentar fallo terapéutico o abandonar el tratamiento por otras causas, en cuyo caso pasan a otra línea de tratamiento (OBA), o lo suspenden definitivamente. Los pacientes con OBA que fallan a este tratamiento pueden seguir a la siguiente línea o abandorar todo tratamiento, quedando con medidas de soporte. El comparador fue la secuencia de tratamiento $\sin \mathrm{M}+\mathrm{S}$, es decir, una en la que los pacientes inician directamente con OBA. Los pacientes, luego de que el tratamiento con OBA falla o lo abandonan, podían recibir terapias adicionales, como neuromodulación sacra o neuroestimulación tibial posterior, o pasar a un estado de incontinencia permanente. La distribución de pacientes entre las distintas alternativas fue definida por expertos clínicos locales.

Parámetros clínicos: los parámetros de eficacia considerados en el modelo fueron los EIUs de urgencia y la frecuencia de micciones por día. La respuesta al tratamiento se basó en la reducción del número diario de EIUs, que puede ser de $50 \%$ o $100 \%$.

Los parámetros de eficacia fueron obtenidos de los metaanálisis desarrollados por Drake et al. ${ }^{18}$ y Kelleher et al. ${ }^{19}$ De estos, se obtuvieron las razones de probabilidades (RPs) y la diferencia promedio de micciones para $\mathrm{M}+\mathrm{S}$ y OBA frente a la terapia con $50 \mathrm{mg}$ de mirabegron en monoterapia (Ver - Tabla 1). En el brazo de $50 \mathrm{mg}$ de mirabegron, $69,0 \%$ de los pacientes tuvieron una reducción del $50 \%$ de los EIUs, y 46,3\%, una reducción del $100 \%{ }^{9}$ Con cada nivel de respuesta, se calculó la nueva distribución de pacientes entre los diferentes niveles de severidad cuando están con tratamiento $\mathrm{M}+$ Sy cuando reciben OBA, y, de esta forma, se obtuvieron costos diferenciales relacionados con el consumo de pañales y, asimismo, en términos de calidad de vida. Se aplicó la distribución de 
Tabla 1 Parámetros del modelo

\begin{tabular}{|c|c|c|c|c|c|c|}
\hline Parámetro & Caso base & Mínimo & Máximo & Distribución & Parámetros & Fuente \\
\hline \multicolumn{7}{|l|}{ Generales } \\
\hline Tasa de descuento & 0,05 & 0,04 & 0,12 & Fija & - & 17 \\
\hline Horizonte de análisis (ciclos) & 20,00 & 20,00 & 20,00 & Fija & - & $13-16$ \\
\hline \multicolumn{7}{|l|}{ Características de los pacientes } \\
\hline EIU (basal) & 3,18 & 0,00 & 6,65 & Normal & $\mu: 3,18, \sigma: 1,66$ & 9 \\
\hline \# de micciones (basal) & 11,19 & 7,92 & 14,46 & Normal & $\mu: 11,19, \sigma: 1,64$ & 9 \\
\hline EIU nivel 1 (0 episodio) - basal & 0,06 & 1,00 & 0,00 & Fija & & 9 \\
\hline EIU nivel 2 (>0-1 episodio) - basal & 0,31 & 0,00 & 0,00 & Fija & & 9 \\
\hline EIU nivel 3 (> 1-2 episodios) - basal & 0,27 & 0,00 & 0,00 & Fija & & 9 \\
\hline EIU nivel 4 (> 2-3 episodios) - basal & 0,19 & 0,00 & 0,00 & Fija & & 9 \\
\hline EIU nivel 5 (> 3 episodios) - basal & 0,16 & 0,00 & 1,00 & Fija & & 9 \\
\hline \multicolumn{7}{|l|}{ Parámetros clínicos } \\
\hline $\begin{array}{l}\text { Respuesta de } \mathrm{M}+\mathrm{S} \text { versus } 50 \mathrm{mg} \text { de } \\
\text { mirabegron }-50 \%\end{array}$ & 1,48 & 1,24 & 1,72 & Normal & $\mu: 1,48, \sigma: 0,12$ & 19 \\
\hline $\begin{array}{l}\text { Respuesta de OBA versus } 50 \mathrm{mg} \text { de } \\
\text { mirabegron }-50 \%\end{array}$ & 2,08 & 1,39 & 3,13 & Normal & $\mu: 2,08, \sigma: 0,43$ & 18 \\
\hline $\begin{array}{l}\text { Respuesta de } \mathrm{M}+\mathrm{S} \text { versus } 50 \mathrm{mg} \text { de } \\
\text { mirabegron }-100 \%\end{array}$ & 1,58 & 1,35 & 1,81 & Normal & $\mu: 1,58, \sigma: 0,12$ & 19 \\
\hline $\begin{array}{l}\text { Respuesta de OBA versus } 50 \mathrm{mg} \text { de } \\
\text { mirabegron }-100 \%\end{array}$ & 3,03 & 2,04 & 4,55 & Normal & $\mu: 3,03, \sigma: 0,63$ & 18 \\
\hline Cambio en micciones con $\mathrm{M}+\mathrm{S}$ & $-0,59$ & $-0,30$ & $-0,88$ & Beta & $\alpha: 26,92, \beta:-72,53$ & 18 \\
\hline Cambio en micciones con OBA & $-0,72$ & $-0,36$ & $-1,08$ & Beta & $\alpha: 28,24, \beta:-67,46$ & 18 \\
\hline Persistencia $\mathrm{M}+\mathrm{S}$ (12 meses) & 0,43 & 0,12 & 0,66 & Beta & $\alpha: 5,40, \beta: 7,15$ & 21,26 \\
\hline Persistencia OBA (12 meses) & 0,93 & 0,74 & 1,00 & Beta & $\alpha: 13,77, \beta: 1,02$ & 22 \\
\hline Persistencia OBA (24 meses) & 0,73 & 0,58 & 0,87 & Beta & $\alpha: 26,45, \beta: 9,87$ & 22 \\
\hline Persistencia OBA (36 meses) & 0,59 & 0,47 & 0,71 & Beta & $\alpha: 40,41, \beta: 28,08$ & 22 \\
\hline Persistencia OBA (42 meses) & 0,51 & 0,41 & 0,62 & Beta & $\alpha: 48,23, \beta: 45,88$ & 22 \\
\hline $\begin{array}{l}\text { Pacientes en los que fallan la OBA y } \\
\text { reciben tercera línea de tratamiento }\end{array}$ & 0,50 & 0,00 & 1,00 & Beta & $\alpha: 1,5, \beta: 1,5$ & Expertos \\
\hline $\begin{array}{l}\text { Pacientes en los que fallan el } \mathrm{M}+\mathrm{Sy} \\
\text { no reciben OBA }\end{array}$ & 0,18 & 0,00 & 0,22 & Beta & $\alpha: 8,93, \beta: 40,68$ & Expertos \\
\hline \multicolumn{7}{|l|}{$\begin{array}{l}\text { Costos de medicación y } \\
\text { monitorización }\end{array}$} \\
\hline Dosis diaria de mirabegron & $50 \mathrm{mg}$ & $25 \mathrm{mg}$ & $50 \mathrm{mg}$ & Uniforme & $\alpha: 25, \beta: 50$ & 7 \\
\hline Dosis diaria de solifenacina & $5 \mathrm{mg}$ & $5 \mathrm{mg}$ & $10 \mathrm{mg}$ & Uniforme & $\alpha: 5, \beta: 10$ & 7 \\
\hline Precio por mg de mirabegron & 84 & 67 & 101 & Gamma & $\alpha: 151,8, \beta: 0,55$ & 27 \\
\hline Precio por mg de solifenacina & 466 & 373 & 560 & Gamma & $\alpha: 155,53, \beta: 3$ & 27 \\
\hline $\begin{array}{l}\text { Precio por mg de toxina botulínica } \\
\text { (OBA) }\end{array}$ & 4,150 & 3,429 & 5,720 & Gamma & $\alpha: 82,03, \beta: 50,59$ & 27 \\
\hline $\begin{array}{l}\text { Costo de administración de la OBA } \\
\text { (\$/aplicación) }\end{array}$ & 189,514 & 182,225 & $1,588,631$ & Gamma & $\alpha: 1,16, \beta: 163.079,79$ & 23 \\
\hline $\begin{array}{l}\text { Intervalo entre aplicaciones de la } \\
\text { OBA (meses) }\end{array}$ & 7,60 & 6,00 & 18,00 & Uniforme & $\alpha: 6, \beta: 18$ & 22 \\
\hline Costo de monitorización del $\mathrm{M}+\mathrm{S}$ & 55,289 & 53,163 & 62,944 & Gamma & $\alpha: 798,68, \beta: 69,23$ & 23,27 \\
\hline Costo de monitorización de la OBA & 74,510 & 71,589 & 85,497 & Gamma & $\alpha: 717,70, \beta: 103,83$ & 23,27 \\
\hline
\end{tabular}


Tabla 1 (Continued)

\begin{tabular}{|c|c|c|c|c|c|c|}
\hline Parámetro & Caso base & Mínimo & Máximo & Distribución & Parámetros & Fuente \\
\hline \multicolumn{7}{|l|}{ Costo de insumos (pañales) } \\
\hline Uso pañales - nivel 1 & 0,23 & 0,19 & 0,27 & Normal & $\mu: 0,23, \sigma: 0,02$ & 16 \\
\hline Uso pañales - nivel 2 & 0,78 & 0,69 & 0,87 & Normal & $\mu: 0,78, \sigma: 0,04$ & 16 \\
\hline Uso pañales - nivel 3 & 1,48 & 1,33 & 1,64 & Normal & $\mu: 1,48, \sigma: 0,08$ & 16 \\
\hline Uso pañales - nivel 4 & 1,94 & 1,74 & 2,14 & Normal & $\mu: 1,94, \sigma: 0,1$ & 16 \\
\hline Uso pañales - nivel 5 & 4,04 & 3,73 & 4,36 & Normal & $\mu: 4,04, \sigma: 0,16$ & 16 \\
\hline Costo de pañales (\$/unidad) & 2,785 & 2,213 & 3,200 & Gamma & $\alpha: 198,89, \beta: 14$ & 24,25 \\
\hline \multicolumn{7}{|l|}{$\begin{array}{l}\text { Frecuencia y costo de reacciones } \\
\text { adversas }\end{array}$} \\
\hline Boca seca $-M+S$ & $6,1 \%$ & $6,1 \%$ & $6,1 \%$ & Fija & & 21 \\
\hline Constipación - M+S & $2,8 \%$ & $2,8 \%$ & $2,8 \%$ & Fija & & 21 \\
\hline Visión borrosa - M+S & $0,4 \%$ & $0,4 \%$ & $0,4 \%$ & Fija & & 21 \\
\hline Hipertensión - M + S & $7,6 \%$ & $7,6 \%$ & $7,6 \%$ & Fija & & 21 \\
\hline Retención urinaria $-\mathrm{M}+\mathrm{S}$ & $0,6 \%$ & $0,6 \%$ & $0,6 \%$ & Fija & & 21 \\
\hline Retención urinaria - OBA & $6,0 \%$ & $6,0 \%$ & $6,0 \%$ & Fija & & 21 \\
\hline Infección del tracto urinario $-\mathrm{M}+\mathrm{S}$ & $4,0 \%$ & $4,0 \%$ & $4,0 \%$ & Fija & & 21 \\
\hline Infección del tracto urinario - OBA & $18,0 \%$ & $18,0 \%$ & $18,0 \%$ & Fija & & 21 \\
\hline Taquicardia $-\mathrm{M}+\mathrm{S}$ & $0,9 \%$ & $0,9 \%$ & $0,9 \%$ & Fija & & 21 \\
\hline Disuria - OBA & $9,0 \%$ & $9,0 \%$ & $9,0 \%$ & Fija & & 21 \\
\hline Bacteriuria - OBA & $4,0 \%$ & $4,0 \%$ & $4,0 \%$ & Fija & & 21 \\
\hline $\begin{array}{l}\text { Volumen postmiccional elevado que } \\
\text { no requiere cateterización - OBA }\end{array}$ & $3,0 \%$ & $3,0 \%$ & $3,0 \%$ & Fija & & 21 \\
\hline Costo RAM M + S & 27,530 & 25,425 & 33,528 & Gamma & $\alpha: 415,59, \beta: 66,24$ & 23,27 \\
\hline Costo RAM OBA & 40,504 & 37,159 & 49,047 & Gamma & $\alpha: 1160,73, \beta: 34,9$ & 23,27 \\
\hline \multicolumn{7}{|l|}{$\begin{array}{l}\text { Costo de intervenciones tras la } \\
\text { OBA }\end{array}$} \\
\hline Costo tras la OBA & 743,807 & 726,185 & 807,980 & Gamma & $\alpha: 2976,89, \beta: 249,86$ & 23,27 \\
\hline \multicolumn{7}{|l|}{ Utilidades } \\
\hline Basal & 0,78 & 0,63 & 0,94 & Uniforme & $\alpha: 0,63, \beta: 0,94$ & 13 \\
\hline ElUs nivel $1-0$ & 0,06 & 0,05 & 0,07 & Uniforme & $\alpha: 0,05, \beta: 0,07$ & 13 \\
\hline EIUs nivel 2->0-1 & 0,04 & 0,03 & 0,05 & Uniforme & $\alpha: 0,03, \beta: 0,05$ & 13 \\
\hline ElUs nivel 3-> 1-2 & 0,03 & 0,03 & 0,04 & Uniforme & $\alpha: 0,03, \beta: 0,04$ & 13 \\
\hline ElUs nivel 4-> 2-3 & 0,01 & 0,01 & 0,02 & Uniforme & $\alpha: 0,01, \beta: 0,02$ & 13 \\
\hline ElUs nivel 5->3 & 0,00 & 0,00 & 0,00 & Uniforme & $\alpha: 0, \beta: 0$ & 13 \\
\hline Micciones nivel $1-<8$ & 0,06 & 0,05 & 0,08 & Uniforme & $\alpha: 0,05, \beta: 0,08$ & 13 \\
\hline Micciones nivel 2-> 8-10 & 0,04 & 0,03 & 0,05 & Uniforme & $\alpha: 0,03, \beta: 0,05$ & 13 \\
\hline Micciones nivel 3-> 10-12 & 0,02 & 0,02 & 0,02 & Uniforme & $\alpha: 0,02, \beta: 0,02$ & 13 \\
\hline Micciones nivel 4-> 12-14 & 0,01 & 0,01 & 0,01 & Uniforme & $\alpha: 0,01, \beta: 0,01$ & 13 \\
\hline Micciones nivel 5->14 & 0,00 & 0,00 & 0,00 & Uniforme & $\alpha: 0, \beta: 0$ & 13 \\
\hline
\end{tabular}

Abreviaturas: EIU, episodio de incontinencia unriunaria; $M+S$, régimen combinado de mirabegron y solifenacina; OBA, onabotulinumtoxina A; RAM, reacciones adversas a medicamentos.

Poisson para calcular la distribución de pacientes en cada nivel de frecuencia de micciones, asumiendo que todos presentan el cambio promedio reportado en el metaanálisis de Drake et al. ${ }^{18}$ Se asumió que los pacientes que suspenden el tratamiento farmacológico en la secuencia $\mathrm{M}+\mathrm{S}$ vuelven a su estado inicial, es decir, a los niveles basales de EIUs y micciones, que corresponden a los parámetros iniciales del brazo control de mirabegron del estudio SYNERGY. ${ }^{20}$

La información de continuidad del tratamiento con el régimen combinado se basó en la información de 
persistencia reportada en el estudio de Yeowell et al. ${ }^{21}$ Para OBA, se consideró la información proveniente de un estudio de extensión de seguimiento a largo plazo (3,5 años). ${ }^{22} \mathrm{Se}$ extrapoló como dato de persistencia la cantidad de pacientes aun enrolados en el estudio a los años 1 (781), 2 (611), 3 (495) y 3,5 (430), de los 839 que iniciaron.

Para el modelo, se tuvo en cuenta que no todos los pacientes en los que falla el tratamiento $\mathrm{M}+\mathrm{S}$ reciben tratamiento con OBA, y, asimismo, no todos los pacientes en los que falla la OBA reciben una tercera línea de tratamiento. La proporción en cada caso fue obtenida de expertos clínicos locales.

Desenlaces: las medidas de efectividad consideradas fueron los años de vida ganados y los años de vida ganados ajustados por calidad (AVACs). Los AVACs son la medida principal, pues permiten capturar el impacto de las tecnologías sobre la calidad de vida relacionada con la salud, y son la métrica recomendada por el IETS para evaluaciones económicas en nuestro medio. ${ }^{17}$ También se realizó un análisis de la costoefectividad incremental, tomando como medida de efectividad el número de respondedores (paciente-año) en cada secuencia de tratamiento.

Los valores de utilidad para los estados de salud fueron independientes del tratamiento. Las medidas de utilidad fueron diferenciales según la severidad de los EIUs y la frecuencia de micciones diarias. La calidad de vida relacionada con la salud de los diferentes estados fue evaluada con el cuestionario European Quality of Life-5 Dimensions (EQ-5D), pero todos los estudios considerados hacen referencia a otras regiones. No se aplicaron pérdidas de utilidad asociadas con el perfil de seguridad. Para el modelo, se consideró la ecuación de estimación para utilidades definida en Aballéa et al. ${ }^{13}$ Los coeficientes de la ecuación de regresión empleados para estimar las utilidades a partir de síntomas urinarios pueden ser consultados en el - Material Suplementario (solamente online).

Supuestos del modelo: en el modelo, se tiene en cuenta que el paciente puede descontinuar el tratamiento bien por fallo terapéutico o por otras causas, que incluyen el perfil de seguridad. En el modelo, también se tiene en cuenta que, luego de fallo terapéutico con $\mathrm{M}+\mathrm{S}$ u OBA, cierta proporción de pacientes puede no recibir ninguna intervención, en cuyo caso se considera que permanecen con síntomas no controlados hasta el final del horizonte de análisis. No se tuvo en cuenta el riesgo de las comorbilidades en los no respondedores. En ellos, se asumió la misma magnitud de cambio en la frecuencia de micciones para toda cohorte, independientemente del nivel de frecuencia basal.

Estimación de costos: dada la perspectiva del estudio, sólo se tuvieron en cuenta los costos médicos directos. Todos los costos fueron expresados en pesos colombianos (COP) de 2019 (1 USD $=\$ 3.231$ COP). Se incluyeron costos de adquisición, administración, monitorización, insumos, manejo de los eventos adversos, y el de las intervenciones tras la OBA. La metodología empleada para el costeo fue la del caso base, por lo que se consultó a un grupo de expertos para identificar y cuantificar los recursos requeridos para los estados de salud y aquellos diferenciales entre la intervención y el comparador. La información de precios de medicamentos fue obtenida del reporte de 2020 del Sistema de Información de Precios de Medicamentos (Sismed), ${ }^{27}$ y las circulares de regulación de precios de medicamentos emitidas por el Ministerio de Salud y Protección Social (MSPS). ${ }^{27}$ Los costos de consultas, laboratorios y procedimientos fueron extraídos del manual tarifario de 2001 del Instituto de Seguros Sociales ${ }^{23}$ (ISSS) más un incremento del $30 \%$.

El esquema de dosificación del régimen combinado se basó en las dosis reportadas en el estudio SYNERGY. ${ }^{9}$ Para la toxina botulínica, se consideró la dosis reportada en otra evaluación económica ${ }^{15}$ desarrollada en Colombia (100 UI/ aplicación). Se empleó un intervalo entre administraciones de 7,6 meses, según lo reportado en la literatura. ${ }^{22}$ El costo de administración de la toxina botulínica código único de procedimientos en salud 861411 (CUPS 261411) fue de $\$ 189,514$ según el manual del ISSS $^{24}+30 \%$. En el análisis de sensibilidad, se tuvo en cuenta un costo de administración de $\$ 1.588,631$, considerando los códigos 999239 para cistoscopia, y 999472F para inyección de toxina botulínica.

Los costos de monitorización incluyeron principalmente las consultas para evaluar la respuesta al tratamiento. En el - Material suplementario (solamente online), se presenta el detalle el uso de los recursos requeridos.

La utilización de pañales/compresas fue diferencial entre los distintos niveles de severidad de la incontinencia, y se basó en otra evaluación de costo-utilidad previamente realizada. ${ }^{16}$ La información de precio de los pañales/compresas fue obtenida de licitaciones públicas, y se consideró un precio promedio de $\$ 2.785$ (rango: 2.213-3.200). ${ }^{24,25}$

La incidencia de eventos adversos se basó en el estudio de Yeowell et al. ${ }^{21}$ La estimación del costo de cada evento siguió la metodología del caso base, descrita anteriormente. ${ }^{17}$

Para las intervenciones luego del fallo de la OBA, se calculó un costo promedio ponderado por la proporción de pacientes que pasan a incontinencia permanente, o reciben neuromodulación sacra o neuroestimulación tibial posterior. En el -Material suplementario (solamente online), se presenta el detalle de los recursos y costos tenidos en cuenta para los estados de salud y los eventos adversos considerados.

En la - Tabla 1 se detallan los parámetros empleados en el modelo.

Análisis de costoefectividad: se calculó la razón de costoefectividad incremental (RCEI) de la tecnología evaluada con respecto al comparador. Se considera que la intervención es costoefectiva si la RCEI está por debajo de 1 vez el producto interno bruto (PIB) per cápita, y como potencialmente costoefectiva si es inferior a 3 veces el PIB per cápita $(\$ 56.005,110)$. En el análisis que considera el número de respondedores como medida de efectividad, la RCEI corresponde al costo de la intervención por respondedor adicional. Con el fin de evaluar la robustez de los resultados obtenidos, se realizaron análisis de sensibilidad determinísticos y probabilísticos, variando los principales parámetros del modelo. 
Tabla 2 Resultados del caso base

Criterio de respuesta: reducción de $50 \%$ en los episodios de incontinencia urinaria

\begin{tabular}{|c|c|c|c|c|c|c|}
\hline Alternativas & Costos & AVACs & $\Delta$ Costos & $\triangle$ AVACs & RCEI & BMN \\
\hline Con $\mathrm{M}+\mathrm{S}$ & $8.372,176$ & 2,87 & \multirow[t]{2}{*}{$1.415,646$} & \multirow[t]{2}{*}{0,10} & \multirow[t]{2}{*}{$13.637,184$} & \multirow[t]{2}{*}{$4.398,120$} \\
\hline $\operatorname{Sin} M+S$ & $6.956,530$ & 2,77 & & & & \\
\hline \multicolumn{7}{|c|}{ Criterio de respuesta: reducción de $100 \%$ en los episodios de incontinencia urinaria } \\
\hline Alternativas & Costos & AVACs & $\Delta$ Costos & $\triangle$ AVACs & RCEI & BMN \\
\hline Con $\mathrm{M}+\mathrm{S}$ & $8.166,512$ & 2,91 & \multirow[t]{2}{*}{$1.749,714$} & \multirow[t]{2}{*}{0,06} & \multirow[t]{2}{*}{$29.313,848$} & \multirow[t]{2}{*}{$1.593,174$} \\
\hline $\operatorname{Sin} M+S$ & $6.416,798$ & 2,85 & & & & \\
\hline
\end{tabular}

Abreviaturas: AVACs, años de vida ajustados por calidad; BMN, beneficio monetario neto; $M+S$, régimen combinado de mirabegron y solifenacina; RCEI, razón de costo-efectividad incremental; BMN beneficio monetario neto.

\section{Resultados}

Para el caso base ( - Tabla 2), la secuencia con $\mathrm{M}+\mathrm{S}$ se asoció con más incremento en el número de AVACs y en los costos que la secuencia sin $\mathrm{M}+\mathrm{S}$; así, se obtuvo una RCEI de \$ $13.637,184$, si se considera el desenlace de reducción de EIUs de $50 \%$, y de $\$ 29.313 .848$, si se considera el del $100 \%$. Esto implica que, bajo el umbral de los tres PIB per cápita informalmente adoptado para Colombia (\$56.005.110), el esquema de tratamiento $\mathrm{M}+\mathrm{S}$ sería costoefectivo en comparación con un esquema de tratamiento $\sin \mathrm{M}+\mathrm{S}$. Al considerar los respondedores como medida de efectividad, se tiene que el costo de la secuencia $\mathrm{M}+\mathrm{S}$ por respondedor adicional es de $\$ 17.035,121$ para el nivel de respuesta de $50 \%$, y de $\$ 11.347,702$ para el del $100 \%$.

Los resultados de los análisis de sensibilidad univariados se muestran en las - Figuras $\mathbf{2}$ y $\mathbf{3}$. Para el nivel de respuesta de $50 \%$, los parámetros que más tuvieron impacto sobre los resultados finales fueron la proporción de pacientes en los que falla la OBA y reciben una tercera línea, los costos de administración de la OBA, y la persistencia al tratamiento
$\mathrm{M}+\mathrm{S}$ a los 12 meses. Sin embargo, ningún cambio en las variables considerado modificó el resultado del caso base.

Para el nivel de respuesta del $100 \%$, los parámetros que más tuvieron impacto sobre los resultados finales fueron el costo de administración de la OBA, la distribución inicial de los pacientes, y la persistencia de $\mathrm{M}+\mathrm{S}$ (a los 12 meses).

En el -Material suplementario (solamente online), se muestran los resultados del análisis de sensibilidad probabilístico. En el 97,6\% y el 82,9\% de las simulaciones, la secuencia $\mathrm{M}+\mathrm{S}$ fue costoefectiva para un nivel de respuesta de $50 \%$ y $100 \%$, respectivamente. La probabilidad de que el esquema de tratamiento $\mathrm{M}+\mathrm{S}$ sea costoefectivo con la disposición a pagar del sistema de salud colombiano es de $92,5 \%$ si se considera un nivel de respuesta de $50 \%$, y de $78,2 \%$ si se considera un nivel de respuesta de $100 \%$.

\section{Discusión}

De acuerdo con los resultados de la evaluación económica, el tratamiento $\mathrm{M}+\mathrm{S}$ presenta mayor efectividad, pues proporciona más AVACs que la secuencia de tratamiento

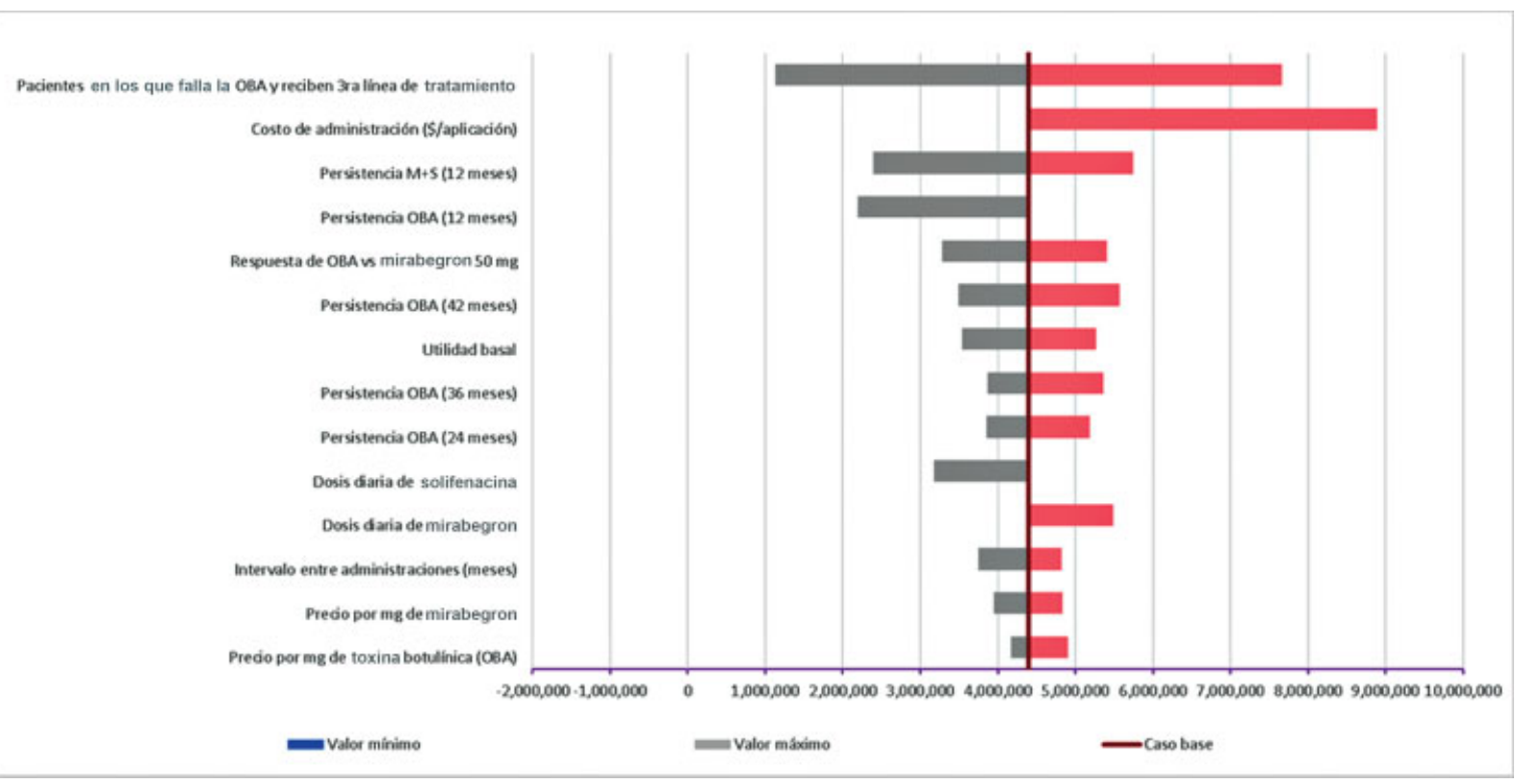

Fig. 2 Resultados del análisis de sensibilidad determinístico - reducción del 50\% en los ElUs. 


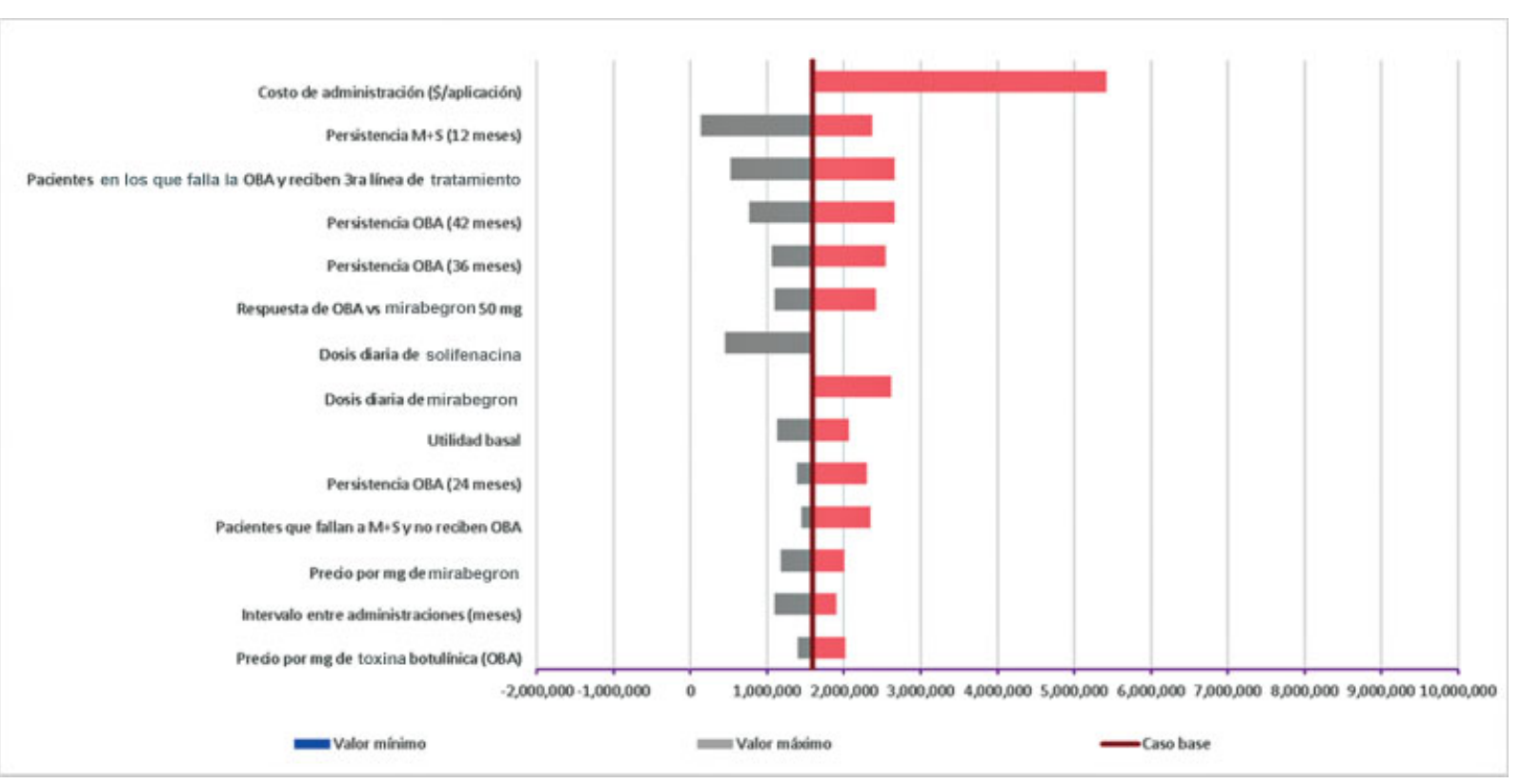

Fig. 3 Resultados del análisis de sensibilidad determinístico - Reducción EIU 100\%

sin la combinación, y, desde el punto de vista del Sistema General de Seguridad Social en Salud, resulta una alternativa altamente costoefectiva para un nivel de respuesta de $50 \%$ (reducción de EIUs/día de 50\%).

Para un nivel de respuesta de 50\%, la variable que afecta el resultado del $\mathrm{M}+\mathrm{S}$ de manera más sustancial es la cantidad de pérdidas de tratamiento después de fallo de la $\mathrm{M}+\mathrm{S}$ (es decir, pacientes que no pasan a OBA, sino que permanecen sintomáticos). Cuando esta supera el $18 \%$, la secuencia $M+S$ deja de ser costoefectiva. Los resultados fueron robustos a cambios en las tasas de descuento o el uso de las dosis máximas recomendadas para los medicamentos.

No se identificaron evaluaciones económicas en las que se realizara una comparación de $\mathrm{M}+\mathrm{S}$ frente a secuencias que inician directamente con OBA luego de fallo del tratamiento farmacológico. La mayoría de las evaluaciones corresponden a comparaciones entre la combinación y monoterapias, en las que se han identificado resultados favorables en términos de costoefectividad. La validez externa del presente análisis es limitada debido a las diferencias en los esquemas de financiación de medicamentos, los costos de los servicios de salud, y los precios de los medicamentos entre regiones.

Las limitaciones de este estudio se relacionan con los supuestos aplicados y la falta de disponibilidad de información local relacionada con persistencia. Para el análisis, se tuvo en cuenta una determinada distribución entre los niveles de severidad de los EIUs, que se basó en el estudio SYNERGY; ${ }^{9}$ sin embargo, los niveles de respuesta podrían diferir entre los distintos niveles de severidad, por lo que los resultados deben interpretarse con precaución.

En cuanto a los valores de utilidad, estos se basaron en otra evaluación económica internacional ${ }^{16}$, pues no se dispone de estudios de calidad de vida en nuestro país, lo cual puede no reflejar las preferencias de los pacientes colombianos. El costeo de los estados de salud y de los eventos adversos se realizó con la ayuda de expertos clínicos, por lo que pueden diferir de la realidad nacional.

\section{Conclusiones}

Los resultados de esta evaluación indican que, para un horizonte de análisis de cinco años, la secuencia de tratamiento $\mathrm{M}+\mathrm{S}$ produce un mayor número de AVACs respecto a las secuencias sin la combinación, y es una alternativa costoefectiva si se considera un umbral de 3 PIB per cápita. Los resultados son robustos a cambios en las tasas de descuento, posología y precios. Entre las variables que tienen mayor impacto sobre los resultados, se encuentra la proporción de pacientes que pueden ser candidatos a la OBA luego del fallo terapéutico de la $M+S$, los costos de administración de la OBA, y la persistencia con la combinación.

\section{Conflicto de Intereses}

Este estudio fue financiado por Astellas Farma Colombia S. A.S. Sin embargo, el análisis y la presentación de los resultados no fueron dictados ni influenciados por Astellas. Los autores declaran no tener ningún otro conflicto de intereses.

\section{Referencias}

1 White N, Iglesia CB. Overactive Bladder. Obstet Gynecol Clin North Am 2016;43(01):59-68. Doi: 10.1016/j.ogc.2015.10.002

2 Marcelissen T, Cornu JN, Antunes-Lopes T, et al. Management of idiopathic overactive bladder syndrome: What is the optimal strategy after failure of conservative treatment? Eur Urol Focus 2018;4(05):760-767. Doi: 10.1016/j.euf.2018.05.004

3 Leron E, Weintraub AY, Mastrolia SA, Schwarzman P. Overactive bladder syndrome: Evaluation and management. Curr Urol 2018; 11(03):117-125. Doi: 10.1159/000447205 
4 Truzzi JC, Gomes CM, Bezerra CA, et al. Overactive bladder - 18 years - Part I. Int Braz J Urol 2016;42(02):188-198. Doi: 10.1590/S16775538.IBJU.2015.0365

5 Plata M, Bravo-Balado A, Robledo D, et al. Prevalence of lower urinary tract symptoms and overactive bladder in men and women over 18 years old: The Colombian overactive bladder and lower urinary tract symptoms (COBaLT) study. Neurourol Urodyn 2019;38(01):200-207. Doi: 10.1002/nau.23828

6 Invima. Acta $\mathrm{N}^{\circ} 1$ de 2020. Comisión revisora. Sala especializada de moléculas nuevas, nuevas indicaciones y medicamentos biológicos. https://www.invima.gov.co/documents/20143/1675737/Acta-No01-de-2020-SEMNNIMB.pdf Published 2020. Accessed June 1, 2020.

7 U.S. Food and Drug Administration (FDA) Summary products characteristicas: Mirabegron (Myrbetriq). https://www. accessdata.fda.gov/drugsatfda_docs/label/2018/202611s011lbl. pdf Published 2012. Accessed June 1, 2020.

8 Abrams P, Kelleher C, Staskin D, et al. Combination treatment with mirabegron and solifenacin in patients with overactive bladder: exploratory responder analyses of efficacy and evaluation of patient-reported outcomes from a randomized, double-blind, factorial, dose-ranging, Phase II study (SYMPHONY). World J Urol 2017;35(05):827-838. Doi: 10.1007/s00345-016-1908-1

9 Herschorn S, Chapple CR, Abrams P, et al. Efficacy and safety of combinations of mirabegron and solifenacin compared with monotherapy and placebo in patients with overactive bladder (SYNERGY study). BJU Int 2017;120(04):562-575. Doi: 10.1111/ bju. 13882

10 Drake MJ, Chapple C, Esen AA, et al; BESIDE study investigators. Efficacy and Safety of Mirabegron Add-on Therapy to Solifenacin in Incontinent Overactive Bladder Patients with an Inadequate Response to Initial 4-Week Solifenacin Monotherapy: A Randomised Double-blind Multicentre Phase 3B Study (BESIDE). Eur Urol 2016;70(01):136-145. Doi: 10.1016/j.eururo.2016.02.030

11 Peng L, Zeng X, Shen H, Luo D-Y. Efficacy and safety of combinations of mirabegron and solifenacin in patients with overactive bladder: a systematic review and meta-analysis. Int J Clin Exp Med 2019;12(02):1355-1365

12 Instituto Nacional de Vigilancia de Medicamentos y Alimentos Invima. Consulta de Registros Sanitarios. http://consultaregistro. invima.gov.co:8082/Consultas/consultas/consreg_encabcum.jsp Published 2019. Accessed May 27, 2018.

13 Aballéa S, Maman K, Thokagevistk K, et al. Cost effectiveness of mirabegron compared with tolterodine extended release for the treatment of adults with overactive bladder in the United Kingdom. Clin Drug Investig 2015;35(02):83-93. Doi: 10.1007/ s40261-014-0240-z

14 Bertapelle MP, Vottero M, Popolo GD, et al. Sacral neuromodulation and Botulinum toxin $A$ for refractory idiopathic overactive bladder: a cost-utility analysis in the perspective of Italian Healthcare System. World J Urol 2015;33 (08):1109-1117. Doi: 10.1007/s00345-014-1401-7

15 Castaño J, Lopera A, Orozco J, Valencia J. Cost utility analysis of sacral neuromodulation versus botox $a$ in the treatment of overactive bladder in Colombia. Value Health 2014;17(03): A294. Doi: 10.1016/j.jval.2014.03.1710

16 Hakimi Z, Kelleher C, Aballéa S, et al. Cost-effectiveness of solifenacin compared with oral antimuscarinic agents for the treatment of patients with overactive bladder in the UK. J Mark Access Health Policy 2018;6(01):1438721. Doi: 10.1080/ 20016689.2018.1438721

17 Instituto de Evaluación Tecnológica en Salud. Manual para la elaboración de evaluaciones económicas en Salud. Bogotá D.C.: IETS; 2014

18 Drake MJ, Nitti VW, Ginsberg DA, et al. Comparative assessment of the efficacy of onabotulinumtoxinA and oral therapies (anticholinergics and mirabegron) for overactive bladder: a systematic review and network meta-analysis. BJU Int 2017; 120(05):611-622. Doi: 10.1111/bju.13945

19 Kelleher C, Hakimi Z, Zur R, et al. Efficacy and tolerability of mirabegron compared with antimuscarinic monotherapy or combination therapies for overactive bladder: A systematic review and network meta-analysis. Eur Urol 2018;74(03): 324-333. Doi: 10.1016/j.eururo.2018.03.020

20 Allison SJ, Gibson W. Mirabegron, alone and in combination, in the treatment of overactive bladder: real-world evidence and experience. Ther Adv Urol 2018;10(12):411-419. Doi: 10.1177/1756287218801282

21 Yeowell G, Smith P, Nazir J, Hakimi Z, Siddiqui E, Fatoye F. Realworld persistence and adherence to oral antimuscarinics and mirabegron in patients with overactive bladder (OAB): a systematic literature review. BMJ Open 2018;8(11):e021889. Doi: 10.1136/bmjopen-2018-021889

22 Nitti VW, Ginsberg D, Sievert K-D, et al;191622-096 Investigators. Durable efficacy and safety of long-term onabotulinumtoxinA treatment in patients with overactive bladder syndrome: Final results of a 3.5-year study. J Urol 2016;196(03):791-800. Doi: $10.1016 /$ j.juro.2016.03.146

23 Instituto de Seguros Sociales. Acuerdo 256 de 2001. Manual de tarifas de la Entidad Promotora de Salud EPS-ISS. https:// lexsaludcolombia.files.wordpress.com/2010/10/tarifas-iss-2001. pdf. Published 2001. Accessed June 4, 2020.

24 Detalle licitación SECOP II: Sistema de Salud de la Policía Nacional. https://construyored.com/licitaciones/colombia/258726580 Published 2019. Accessed November 26, 2019.

25 Licitación pública: Subred integrada de Servicios de Salud Sur E.S. E. https://construyored.com/licitaciones/colombia/258726580 Published 2017. Accessed November 26, 2019.

26 Kato D, Tabuchi H, Uno S. Three-year safety, efficacy and persistence data following the daily use of mirabegron for overactive bladder in the clinical setting: A Japanese postmarketing surveillance study. Low Urin Tract Symptoms 2019; 11(02):0152-0161. Doi: 10.1111/luts.12237

27 Ministerio de Salud y Protección Social (MSPS) Sismed - Sistema de información de precios de medicamentos. Reporte 2020. http://www.sispro.gov.co/recursosapp/app/Pages/Precios de Medicamentos - Circular 2 de 2012 Excel.aspx. Published 2021. Accessed April 10, 2021. 